\title{
SPECTRAL APPROXIMATION OF PATTERN-FORMING NONLINEAR EVOLUTION EQUATIONS WITH DOUBLE-WELL POTENTIALS OF QUADRATIC GROWTH
}

\author{
NICOLAS CONDETTE, CHRISTOF MELCHER, AND ENDRE SÜLI
}

\begin{abstract}
This paper is concerned with the analysis of a numerical algorithm for the approximate solution of a class of nonlinear evolution problems that arise as $\mathrm{L}^{2}$ gradient flow for the Modica-Mortola regularization of the functional

$$
v \in \mathrm{BV}\left(\mathbb{T}^{d} ;\{-1,1\}\right) \mapsto E(v):=\frac{\gamma}{2} \int_{\mathbb{T}^{d}}|\nabla v|+\frac{1}{2} \sum_{k \in \mathbb{Z}^{d}} \sigma(k)|\hat{v}(k)|^{2} .
$$

Here $\gamma$ is the interfacial energy per unit length or unit area, $\mathbb{T}^{d}$ is the flat torus in $\mathbb{R}^{d}$, and $\sigma$ is a nonnegative Fourier multiplier, that is continuous on $\mathbb{R}^{d}$, symmetric in the sense that $\sigma(\xi)=\sigma(-\xi)$ for all $\xi \in \mathbb{R}^{d}$ and that decays to zero at infinity.

Such functionals feature in mathematical models of pattern-formation in micromagnetics and models of diblock copolymers. The resulting evolution equation is discretized by a Fourier spectral method with respect to the spatial variables and a modified Crank-Nicolson scheme in time. Optimal-order a priori bounds are derived on the global error in the $\ell^{\infty}\left(0, T ; \mathrm{L}^{2}\left(\mathbb{T}^{d}\right)\right)$ norm.
\end{abstract}

\section{INTRODUCTION}

We are interested in pattern-formation phenomena that stem from a competition of interfacial and dipolar energies. More precisely, we consider discrete evolution problems that approximate local minimizers $u: \mathbb{T}^{d} \rightarrow\{ \pm 1\}$ of variational problems on a $2 \pi$-periodic domain $\mathbb{T}^{d}$ (flat torus of dimension $d=2,3$ ) for energy functionals of the form

$$
v \mapsto E(v)=\frac{\gamma}{2} \int_{\mathbb{T}^{d}}|\nabla v|+\frac{1}{2} \sum_{k \in \mathbb{Z}^{d}} \sigma(k)|\hat{v}(k)|^{2} .
$$

Recall that $\frac{1}{2} \int_{\mathbb{T}^{d}}|\nabla v|=\operatorname{Per}\left(\{v=1\} ; \mathbb{T}^{d}\right)$ agrees with the perimeter functional. The parameter $\gamma>0$ is the interfacial energy per unit length or area, for $d=2$ and $d=3$, respectively, and $v \mapsto \mathcal{F} v=\hat{v}: \mathbb{Z}^{d} \rightarrow \mathbb{C}$ denotes the Fourier transform on $\mathbb{T}^{d}$. The Fourier multipliers we are considering are mostly given in terms of nonnegative continuous functions $\sigma \in \mathrm{C}_{0}\left(\mathbb{R}^{d}\right)$ that are symmetric in the sense that $\sigma(\xi)=\sigma(-\xi)$ for all $\xi \in \mathbb{R}^{d}$ and that decay to zero at infinity. Such dipolar interactions prefer, from a variational standpoint, high frequencies and therefore give rise to oscillations. Typical examples include the following:

Received by the editor April 23, 2009 and, in revised form, September 23, 2009.

2010 Mathematics Subject Classification. Primary 65M70; Secondary 82D40, 82D60.

(C)2010 American Mathematical Society Reverts to public domain 28 years from publication 
- magnetic garnet films (with $d=2$ ); cf. [9, 11]:

$$
\sigma(k)=\frac{1-\exp (-\delta|k|)}{\delta|k|} \quad \text { for } \quad k \neq 0 \quad \text { and } \quad \sigma(0)=1
$$

- diblock copolymers (with $d=3$ ); cf. [4]:

$$
\sigma(k)=|k|^{-2} \text { for } \quad k \neq 0,
$$

on the restricted class $\mathcal{A}=\left\{v \in \mathrm{BV}\left(\mathbb{T}^{d} ;\{-1,1\}\right): \int_{\mathbb{T}^{3}} v \mathrm{~d} x=0\right\}$.

In view of specific applications it is interesting to let the Fourier multiplier $\sigma$ depend on a system parameter such as, for example, the relative sample thickness $\delta$ in our first example of a magnetic film, and to study the morphology and bifurcation of patterns when the parameters $\gamma$ and $\delta$ of the two competing energy contributions vary. Parameter studies of this kind are one of the main motivations for performing numerical simulations of physical phenomena for pattern-formation equations.

Our approach is based on a Modica-Mortola approximation for the perimeter functional and the associated $\mathrm{L}^{2}\left(\mathbb{T}^{d}\right)$ gradient flow. Recall that for a nonnegative double-well potential $W$, with wells at \pm 1 and the renormalization

$$
\int_{-1}^{1} \sqrt{2 W(v)} \mathrm{d} v=1
$$

the functional

$$
v \mapsto \gamma \int_{\mathbb{T}^{d}}\left(\frac{\varepsilon}{2}|\nabla v|^{2}+\frac{1}{\varepsilon} W(v)\right) \mathrm{d} x
$$

approximates the interfacial energy in the sense of $\Gamma$-convergence by finite transitionlayers of thickness $\varepsilon>0$; cf. e.g. [1, 2, 5]. Expressing the dipolar energy in units of the typical interfacial energy, i.e., renormalizing the multiplier $\sigma=\sigma(k)$ by $\gamma$, we can assume that $\gamma=1$. Hence our considerations will be based on the energy

$$
v \mapsto E_{\varepsilon}(v):=\int_{\mathbb{T}^{d}}\left(\frac{\varepsilon}{2}|\nabla v|^{2}+\frac{1}{\varepsilon} W(v)\right) \mathrm{d} x+\frac{1}{2} \sum_{k \in \mathbb{Z}^{d}} \sigma(k)|\mathcal{F} v(k)|^{2}
$$

for $\varepsilon>0$ small but fixed and the associated gradient flow with variational formulation

$$
\partial_{t} u+\nabla_{u} E_{\varepsilon}(u)=0
$$

that, in its long-time asymptotics, leads to local minimizers approximating those of the sharp-interface problem. The energy gradient decomposes as

$$
\nabla_{u} E_{\varepsilon}(u)=\mathcal{L}_{\varepsilon} u-\mathcal{N}_{\varepsilon}(u)
$$

where the linear part $\mathcal{L}_{\varepsilon}$ can be represented in terms of the Fourier multiplier $p_{\varepsilon}: k \in \mathbb{Z}^{d} \mapsto p_{\varepsilon}(k)=\varepsilon|k|^{2}+\sigma(k) \in \mathbb{R}_{\geq 0}$ and is therefore, for any $\varepsilon>0$, uniformly second-order elliptic. Thus we consider the nonlinear evolution equation

$$
u_{t}+\mathcal{L}_{\varepsilon} u=\mathcal{N}_{\varepsilon}(u) \quad \text { for } u(t): \mathbb{T}^{d} \rightarrow \mathbb{R} \quad \text { and } t>0
$$

subject to periodic boundary conditions and the initial condition $u(\cdot, 0)=u_{0}(\cdot)$, where $u_{0}$ is a given $2 \pi$-periodic function defined and continuous on $\mathbb{T}^{d}$. Further, for positive $\varepsilon$, let $\mathcal{L}_{\varepsilon}$ and $\mathcal{N}_{\varepsilon}$ denote, respectively, the linear and nonlinear operator defined by

$$
\begin{aligned}
\mathcal{L}_{\varepsilon} v & :=\mathcal{F}^{-1}\left[\left(\varepsilon|k|^{2}+\sigma(k)\right) \mathcal{F} v(k)\right] \\
\mathcal{N}_{\varepsilon}(v) & :=-\mathrm{D} W_{\varepsilon}(v)
\end{aligned}
$$


with $W_{\varepsilon}(\cdot):=\frac{1}{\varepsilon} W(\cdot)$. We shall assume that $W \in \mathrm{C}^{2}(\mathbb{R})$ is a nonnegative doublewell potential, with local minima at \pm 1 , and that $W$ has at most quadratic growth at infinity in the sense that there exist positive real numbers $K$ and $L$ such that

$$
|\mathrm{D} W(v)|^{2} \leq K W(v) \quad \text { and } \quad\left|\mathrm{D}^{2} W(v)\right| \leq L \quad \text { for all } v \in \mathbb{R} .
$$

To prove that, under appropriate assumptions on the regularity of the analytical solution $u$, the error in the $\ell^{\infty}\left(0, T ; \mathrm{L}^{2}\left(\mathbb{T}^{d}\right)\right)$ norm between $u$ and its numerical approximation generated by our spectral Crank-Nicolson method is of the size $\mathcal{O}\left(N^{-\ell}+\Delta t^{2}\right)$, where $N$ is the number of spectral basis functions used, $\Delta t$ is the time step, and $\ell \geq 2$. In Section 4 we shall assume in addition the existence of derivatives of $W$ of order $\ell+1$, such that

$$
\sup _{v \in \mathbb{R}}\left(\left|\mathrm{D}^{3} W(v)\right|+\cdots+\left|\mathrm{D}^{\ell+1} W(v)\right|\right)<\infty .
$$

The existence and uniqueness of the analytical solution $u$ follows from an abstract reasoning. In fact, as $\mathcal{L}_{\varepsilon}: \mathrm{H}^{2}\left(\mathbb{T}^{d}\right) \subset \mathrm{L}^{2}\left(\mathbb{T}^{d}\right) \rightarrow \mathrm{L}^{2}\left(\mathbb{T}^{d}\right)$ is positive and self-adjoint on $\mathrm{L}^{2}\left(\mathbb{T}^{d}\right)$ and hence sectorial, it generates an analytic semi-group $S_{\varepsilon}=S_{\varepsilon}(t)$ on $\mathrm{L}^{2}\left(\mathbb{T}^{d}\right)$. Then, the uniform Lipschitz condition in (1.3) implies, in view of Duhamel's principle, the existence of a unique classical solution $u \in$ $\mathrm{C}\left([0, \infty) ; \mathrm{L}^{2}\left(\mathbb{T}^{d}\right)\right) \cap \mathrm{C}^{1}\left((0, \infty) ; \mathrm{L}^{2}\left(\mathbb{T}^{d}\right)\right)$ with $u(t) \in \mathrm{H}^{2}\left(\mathbb{T}^{d}\right)$ for any $t \geq 0$ for the Cauchy problem

$$
u_{t}+\mathcal{L}_{\varepsilon} u=\mathcal{N}_{\varepsilon}(u), \quad u(0)=u_{0},
$$

with initial datum $u_{0} \in \mathrm{H}^{2}\left(\mathbb{T}^{d}\right)$; cf. [8, 12. Assuming that $W \in \mathrm{C}^{\infty}(\mathbb{R})$ with bounded higher order derivatives, a bootstrap argument yields smoothness; that is, $u \in \mathrm{C}^{\infty}\left((0, \infty) \times \mathbb{T}^{d}\right)$. Note that from the variational structure $\mathcal{L}_{\varepsilon} u-\mathcal{N}_{\varepsilon}(u)=$ $\nabla_{u} E_{\varepsilon}(u)$, where $\nabla_{u}$ denotes the $\mathrm{L}^{2}$ gradient, we have for a classical solution

$$
\int_{0}^{T}\left\|u_{t}(t)\right\|^{2} \mathrm{~d} t+E_{\varepsilon}(u(T))=E_{\varepsilon}(u(0))
$$

for any $T>0$, with $\|\cdot\|$ signifying the $\mathrm{L}^{2}$ norm on $\mathbb{T}^{d}$. The discrete counterpart of this energy equality, satisfied by the sequence of numerical approximations to the analytical solution $u$, is stated in Lemma 3.1 .

The use of a Fourier spectral method for this problem is particularly convenient, for a number of reasons. Above all, the pattern-formation mechanism driven by dipolar energies with Fourier multipliers can be incorporated directly. Moreover, higher-order gradient flows can be implemented easily. In fact, the discussion below applies to any nonlinear evolution equation of the form

$$
u_{t}+\mathcal{F}^{-1}(\Lambda(|k|) \mathcal{F} u(k))=-\mathrm{D} W(u),
$$

subject to periodic boundary conditions, where $\Lambda$ is a nonnegative function with $0 \leq \Lambda(|k|) \leq$ Const. $\left(1+|k|^{2}\right), k \in \mathbb{Z}^{d}$, and $W$ is a nonnegative double-well potential with at most quadratic growth at infinity in the sense of (1.3); in order to reproduce the error bound stated in Section 4 for equation (1.2) in the case of the more general equation (1.5), hypothesis (1.4) should still be assumed.

We shall show that for such nonnegative double-well potentials $W$ with at most quadratic growth at infinity the numerical approximation of the initial boundaryvalue problem, based on our modified Crank-Nicolson approximation in time and Fourier spectral collocation in space, converges unconditionally in the sense that there is no restriction on the size of the time-step, $\Delta t$, in terms of the number of 
Fourier modes, $N$. In particular, in the singularly perturbed version of the problem, with $0<\varepsilon \ll 1$, the size of the time-step $\Delta t$ depends only on the parameter $\varepsilon$ (in a dimensionally consistent fashion). Moreover, if $W$ satisfies (1.3) and (1.4), we shall show that, under appropriate regularity hypotheses on the analytical solution $u$, the numerical method converges at an optimal rate of $\mathcal{O}\left(N^{-s}+(\Delta t)^{2}\right)$ in the $\ell^{\infty}\left(0, T ; \mathrm{L}^{2}\left(\mathbb{T}^{d}\right)\right)$ norm, with an error constant that only depends on $\varepsilon$ and $s$; here $s \geq 2$ is the Sobolev index of the analytical solution $(x, t) \mapsto u(x, t)$ with respect to its spatial variable $x \in \mathbb{T}^{d}$. Finally, we illustrate our analytical results by a series of numerical experiments.

\section{NotATIONS AND PRELIMINARY CONSIDERATIONS}

2.1. The Fourier system. For $N \in \mathbb{N}_{>0}$ we define the set $\mathbb{Z}_{N}:=\{-N, \ldots, N\}$ and denote its $d$-fold Cartesian product by $\mathbb{Z}_{N}^{d}$. Let us consider first the finitedimensional linear subspace of $\mathrm{L}^{2}\left(\mathbb{T}^{d} ; \mathbb{C}\right)$ defined as

$$
\mathrm{S}_{N}:=\operatorname{span}_{\mathbb{C}}\left\{x \in \mathbb{T}^{d} \mapsto \exp (\mathrm{i} k \cdot x) \in \mathbb{C}: k \in \mathbb{Z}_{N}^{d}\right\} .
$$

Since $\operatorname{card}\left(\mathbb{Z}_{N}\right)=2 N+1$, clearly $\operatorname{dim}_{\mathbb{C}}\left(\mathrm{S}_{N}\right)=(2 N+1)^{d}$. We denote by $P_{N}$ : $\mathrm{L}^{2}\left(\mathbb{T}^{d} ; \mathbb{C}\right) \rightarrow \mathrm{S}_{N}$ the orthogonal projection operator based on truncating the Fourier series at the $N$ th term. Here $\mathrm{L}^{2}\left(\mathbb{T}^{d} ; \mathbb{C}\right)$ is the set of all complex-valued squareintegrable $2 \pi$-periodic functions. We also introduce the subspace of $S_{N}$ of realvalued functions

$$
\mathrm{X}_{N}:=\left\{x \in \mathbb{T}^{d} \mapsto \sum_{k \in \mathbb{Z}_{N}^{d}} c(k) \exp (\mathrm{i} k \cdot x) \in \mathrm{S}_{N}: c(-k)=\overline{c(k)}\right\}
$$

with $\operatorname{dim}_{\mathbb{R}}\left(\mathrm{X}_{N}\right)=(2 N+1)^{d}$. Note that $P_{N}: \mathrm{L}^{2}\left(\mathbb{T}^{d} ; \mathbb{R}\right) \rightarrow \mathrm{X}_{N}$.

Motivated by the discussion in [3], we define the following "symmetrized space" as a substitute for the space $\mathrm{S}_{N}$ for certain discretizations:

$$
\Xi_{N}:=\left\{v \in \mathrm{S}_{N}: c(k)=c(l) \text { for } k \sim l\right\}
$$

here the equivalence relation $k \sim l$ means that $k$ and $l$ differ at most in the sign of the components $k_{j}$ and $l_{j}$ with $\left|k_{j}\right|=\left|l_{j}\right|=N$. Whenever the symmetrized space $\Xi_{N}$ is concerned, we adopt the convention that summation is also symmetrized in the sense that terms whose indices are equal to the highest wave numbers, $\pm N$, are multiplied by appropriate powers of $\frac{1}{2}$; cf. [3] for details. In this case we redefine the space $\mathrm{X}_{N}$ by setting

$$
\mathrm{X}_{N}:=\left\{x \in \mathbb{T}^{d} \mapsto \sum_{k \in \mathbb{Z}_{N}^{d}} c(k) \exp (\mathrm{i} k \cdot x) \in \Xi_{N}: c(-k)=\overline{c(k)}\right\},
$$

with the summation sign understood in the above symmetrized sense.

2.2. Discretization and discrete Fourier transform. For $M \in \mathbb{N}_{>0}$, we use the notation $\mathbb{N}_{M}:=\{0,1,2, \ldots, M-1\}$; we denote the $d$-fold Cartesian product of $\mathbb{N}_{M}$ by $\mathbb{N}_{M}^{d}$ and we define the collocation points

$$
x_{j}:=\frac{2 \pi j}{M} \in \mathbb{T}^{d}, \quad j \in \mathbb{N}_{M}^{d},
$$


referred to as the spectral nodes, that will be used in our spatial discretization. For $M=2 N$ or $M=2 N+1$, respectively, and complex-valued functions $v, w \in$ $\mathrm{C}\left(\mathbb{T}^{d} ; \mathbb{C}\right)$, we consider the inner product

$$
\langle v, w\rangle_{N}:=\left(\frac{2 \pi}{M}\right)^{d} \sum_{j \in \mathbb{N}_{M}^{d}} v\left(x_{j}\right) \overline{w\left(x_{j}\right)}, \quad N=N(M):=\left[\frac{M}{2}\right],
$$

and the associated norm $\|\cdot\|_{N}$, defined by

$$
\|v\|_{N}^{2}:=\langle v, v\rangle_{N} .
$$

The discrete Fourier coefficients of a function $v \in \mathrm{C}\left(\mathbb{T}^{d} ; \mathbb{C}\right)$ with respect to the set of collocation points $x_{j}=\frac{2 \pi j}{M}, j \in \mathbb{N}_{M}^{d}$, are

$$
\tilde{v}(k)=\left(\mathcal{F}_{N} v\right)(k):=\frac{1}{M^{d}} \sum_{j \in \mathbb{N}_{M}^{d}} v\left(x_{j}\right) \mathrm{e}^{-\mathrm{i} k \cdot x_{j}}, \quad k \in \mathbb{Z}_{N}^{d} .
$$

The case $M=2 N+1$ is particularly convenient: for all $v, w \in \mathrm{S}_{N}$ we then have that

$$
\langle v, w\rangle_{N}=(v, w)_{\mathrm{L}^{2}\left(\mathbb{T}^{d} ; \mathbb{C}\right)},
$$

and therefore also $\|v\|_{N}=\|v\|:=\|v\|_{\mathrm{L}^{2}\left(\mathbb{T}^{d} ; \mathbb{C}\right)}$ for all $v \in \mathrm{S}_{N}$. In this case the discrete Fourier inversion formula yields

$$
v\left(x_{j}\right)=\left(\mathcal{F}_{N}^{-1} \tilde{v}\right)\left(x_{j}\right):=\sum_{k \in \mathbb{Z}_{N}^{d}} \tilde{v}(k) \mathrm{e}^{\mathrm{i} k \cdot x_{j}}, \quad j \in \mathbb{N}_{M}^{d} .
$$

Note that $\tilde{v}(k)=\frac{1}{(2 \pi)^{d}}\left\langle v, \mathrm{e}^{\mathrm{i} k \cdot(\cdot)}\right\rangle_{N}$; therefore, on $\mathrm{S}_{N}$ the discrete Fourier coefficients $\tilde{v}(k)$ and the exact Fourier coefficients

$$
\hat{v}(k)=\frac{1}{(2 \pi)^{d}} \int_{\mathbb{T}^{d}} v(x) \mathrm{e}^{-\mathrm{i} k \cdot x} \mathrm{~d} x, \quad k \in \mathbb{Z}^{d},
$$

coincide, i.e. $\tilde{v}(k)=\hat{v}(k)$ for all $v \in \mathrm{S}_{N}$ and all $k \in \mathbb{Z}_{N}^{d}$.

In the case of $M=2 N, \mathbb{Z}_{N}^{d}$ is replaced by the $d$-fold cartesian product of the set $\{-N+1, \ldots, N\}$ and the summation sign $\sum_{k \in \mathbb{Z}_{N}^{d}}$ is understood in the above symmetrized sense, with terms whose indices are equal to the highest wave numbers, $\pm N$, being multiplied by appropriate powers of $\frac{1}{2}$.

2.3. Trigonometric interpolation. For $M=2 N+1$, and collocation points $x_{j}$ defined by (2.1), every real-valued function $f: \mathbb{R} \rightarrow \mathbb{R}$ induces a mapping $v \in \mathrm{X}_{N} \mapsto I_{N} f(v) \in \mathrm{X}_{N}$, where

$$
x \in \mathbb{T}^{d} \mapsto I_{N} f(v(x)):=\sum_{j \in \mathbb{N}_{M}^{d}} f\left(v\left(x_{j}\right)\right) \exp \left(\mathrm{i} j \cdot\left(x-x_{j}\right)\right) .
$$

A relevant special case of this is $I_{N} v$, corresponding to $f(s)=s, s \in \mathbb{R}$. Equivalently, for $v \in \mathrm{C}\left(\mathbb{T}^{d} ; \mathbb{C}\right)$, one can define the trigonometric interpolant $I_{N} v \in \mathrm{S}_{\mathrm{N}}$ or $\Xi_{N}$, respectively, of the function $v$ by

$$
\left\langle I_{N} v, w\right\rangle_{N}=\langle v, w\rangle_{N}
$$


for every $w \in \mathrm{S}_{N}$ if $M=2 N+1$ or every $w \in \Xi_{N}$ if $M=2 N$. The existence of a unique $I_{N} v$ in each case is an immediate consequence of the Riesz representation theorem. More explicitly we have, for $M=2 N+1$, the formula

$$
I_{N} v(x)=\sum_{k \in \mathbb{Z}_{N}^{d}} \tilde{v}(k) \mathrm{e}^{\mathrm{i} k \cdot x}, \quad x \in \mathbb{T}^{d},
$$

where $\tilde{v}(k), k \in \mathbb{Z}_{N}^{d}$, are the discrete Fourier coefficients. In the case of $M=2 N$ this formula needs to be symmetrized at the highest wave numbers according to the definition of $\Xi_{N}$; cf. [3] for details. By the Fourier inversion formula we have that $I_{N} v\left(x_{j}\right)=v\left(x_{j}\right)$ for all $j \in \mathbb{N}_{M}^{d}$. In particular, for the restriction to real-valued functions we have

$$
v \in \mathrm{C}\left(\mathbb{T}^{d} ; \mathbb{R}\right) \mapsto I_{N} v \in \mathrm{X}_{N}
$$

For any given $v \in \mathrm{C}\left(\mathbb{T}^{d} ; \mathbb{C}\right)$, the function $I_{N} v$ represents an approximation of $v$. In fact, $I_{N} v$ converges to $v$ in the $\mathrm{L}^{2}\left(\mathbb{T}^{d} ; \mathbb{C}\right)$ norm, i.e.,

$$
\lim _{N \rightarrow \infty}\left\|v-I_{N} v\right\|=0, \quad v \in \mathrm{C}\left(\mathbb{T}^{d} ; \mathbb{C}\right) .
$$

We also define the aliasing error $R_{N} v:=P_{N} v-I_{N} v$, which is $\mathrm{L}^{2}\left(\mathbb{T}^{d} ; \mathbb{C}\right)$-orthogonal to the projection error $v-P_{N} v$ with $P_{N}$ being the $\mathrm{L}^{2}\left(\mathbb{T}^{d} ; \mathbb{C}\right)$-orthogonal projection onto $\mathrm{X}_{N}$. Thus, for $v \in \mathrm{C}\left(\mathbb{T}^{d} ; \mathbb{C}\right)$,

$$
\left\|v-I_{N} v\right\|^{2}=\left\|v-P_{N} v\right\|^{2}+\left\|R_{N} v\right\|^{2} .
$$

For functions $v \in \mathrm{H}^{s}\left(\mathbb{T}^{d} ; \mathbb{C}\right), s \geq 0$, and with $\mathrm{H}^{s}\left(\mathbb{T}^{d} ; \mathbb{C}\right)$ equipped with the norm $\|\cdot\|_{s}$ defined by

we have

$$
\|v\|_{s}:=\left[\sum_{k \in \mathbb{Z}^{d}}\left(1+|k|^{2 s}\right)|\hat{v}(k)|^{2}\right]^{\frac{1}{2}},
$$

$$
\left\|v-P_{N} v\right\| \leq C(s, d) N^{-s}\|v\|_{s}
$$

for all $N \in \mathbb{N}_{>0}$ and, in the supercritical case, $s>d / 2$,

$$
\left\|R_{N} v\right\| \leq C(s, d) N^{-s}\|v\|_{s}
$$

for all $N \in \mathbb{N}_{>0}$. Concentrating on the physically relevant dimensions $d=2,3$ we have, in particular, that

$$
\left\|R_{N} v\right\| \leq C(s, d) N^{-s}\|v\|_{s} \quad \text { and therefore } \quad\left\|v-I_{N} v\right\| \leq C(s, d) N^{-s}\|v\|_{s}
$$

for all $v \in \mathrm{H}^{s}\left(\mathbb{T}^{d} ; \mathbb{C}\right), s \geq 2$, and all $N \in \mathbb{N}_{>0}$.

2.4. Discrete Fourier integral operators. Recall that for a function $m: \mathbb{Z}^{d} \rightarrow$ $\mathbb{C}$ the associated Fourier integral operator $m(\mathrm{D})$, with symbol $m$, is for sufficiently regular functions $v: \mathbb{T}^{d} \rightarrow \mathbb{C}$ defined by

$$
m(\mathrm{D}): v \mapsto \mathcal{F}^{-1}(m \hat{v}) .
$$

Note that if $m$ is symmetric in the sense that $m(-k)=\overline{m(k)}$ for $k \in \mathbb{Z}^{d}$ and $v$ is real-valued, then $m \hat{v}$ shares this symmetry: $(m \hat{v})(-k)=\overline{(m \hat{v})(k)}$ for $k \in \mathbb{Z}^{d}$, and $m(\mathrm{D}) v$ is real-valued. As one of its main properties, the $\mathrm{L}^{2}\left(\mathbb{T}^{d} ; \mathbb{C}\right)$-orthogonal projection operator $P_{N}$ commutes with $m(\mathrm{D})$ for generic functions, i.e.,

$$
P_{N}(m(\mathrm{D}) v)=m(\mathrm{D}) P_{N} v \quad \text { for all sufficiently regular } v
$$


In other words, $\left[P_{N}, m(\mathrm{D})\right] v=0$ where $\left[P_{N}, m(\mathrm{D})\right]:=P_{N} m(\mathrm{D})-m(\mathrm{D}) P_{N}$ is the commutator of $P_{N}$ and $m(\mathrm{D})$. For the interpolation operator $I_{N}$, however, and a generic function $v \in \mathrm{C}^{\infty}\left(\mathbb{T}^{d} ; \mathbb{C}\right),\left[I_{N}, m(\mathrm{D})\right] v \neq 0$ so that an aliasing error needs to be taken into account. For $N \in \mathbb{N}_{>0}$ the corresponding discrete Fourier integral operator on $\mathrm{S}_{N}$ is simply the restriction of $m(\mathrm{D})$ to trigonometric polynomials in $\mathrm{S}_{N}$; the restriction of $m(\mathrm{D})$ to $\mathrm{S}_{N}$, which we still denote by $m(\mathrm{D})$, can be expressed in terms of the discrete Fourier transform only as follows:

$$
m(\mathrm{D}): v \in \mathrm{S}_{N} \mapsto \mathcal{F}_{N}^{-1}(m \tilde{v}) \in \mathrm{S}_{N} .
$$

As in the "continuous" case, if $m$ is symmetric and $v \in \mathrm{X}_{N}$, then $(m \tilde{v})(-k)=$ $\overline{(m \tilde{v})(k)}$ for $k \in \mathbb{Z}_{N}^{d}$, and hence $m(\mathrm{D}) v \in \mathrm{X}_{N}$. Since $\sigma$ is real-valued with $\sigma(k)=$ $\sigma(-k)$, the action of the operator $\mathcal{L}_{\varepsilon}$ on the space $\mathrm{X}_{N}$ given by

$$
\mathcal{L}_{\varepsilon}: v \in \mathrm{X}_{N} \mapsto \mathcal{L}_{\varepsilon} v=\mathcal{F}_{N}^{-1}\left[\left(\varepsilon|\cdot|^{2}+\sigma(\cdot)\right) \tilde{v}(\cdot)\right] \in \mathrm{X}_{N}
$$

is well-defined. In view of (2.2) and Plancherel's theorem, in $\mathrm{L}^{2}\left(\mathbb{T}^{d} ; \mathbb{C}\right)$, we observe that $\mathcal{L}_{\varepsilon}$ is symmetric with respect to the discrete inner product, i.e.,

$$
\left\langle\mathcal{L}_{\varepsilon} v, w\right\rangle_{N}=\left\langle v, \mathcal{L}_{\varepsilon} w\right\rangle_{N} \quad \text { for all } \quad v, w \in \mathrm{X}_{N} .
$$

Finally, we define the energy-norm $\|\cdot\|_{\mathcal{L}_{\varepsilon}}$ on $\mathrm{X}_{N}$ as follows:

$$
\|v\|_{\mathcal{L}_{\varepsilon}}^{2}:=\left\langle\mathcal{L}_{\varepsilon} v, v\right\rangle_{N}=\sum_{k \in \mathbb{Z}_{N}^{d}}\left(\varepsilon|k|^{2}+\sigma(k)\right)|\tilde{v}(k)|^{2}, \quad v \in \mathrm{X}_{N}
$$

With these preliminary considerations, we are now ready to formulate the numerical method.

\section{The Crank-Nicolson scheme}

We begin by stating the spectral Crank-Nicolson discretization of the variational problem (1.2). We shall then show that the scheme is correctly defined by proving the existence and uniqueness of solutions.

3.1. Definition and stability of the Crank-Nicolson scheme. We consider a modification of the Crank-Nicolson scheme based on a first-order difference quotient of the nonlinear potential $W_{\varepsilon}$. With $W_{\varepsilon}$ as above, we set

$$
\mathcal{N}_{\varepsilon}(v, w):=-\frac{W_{\varepsilon}(v)-W_{\varepsilon}(w)}{v-w} \text { for } \quad v \neq w
$$

with

$$
\mathcal{N}_{\varepsilon}(v, v):=\mathcal{N}_{\varepsilon}(v)=-\mathrm{D} W_{\varepsilon}(v) .
$$

Given $T>0$ and $J \in \mathbb{N}_{>0}$, let $\Delta t:=T / J$. Let us further suppose that $u_{0} \in$ $\mathrm{C}\left(\mathbb{T}^{d} ; \mathbb{R}\right)$, and let $u_{N}^{0}:=I_{N} u_{0} \in \mathrm{X}_{N}$ where $N \in \mathbb{N}_{>0}$. For $n=0,1, \ldots, J-1$, the numerical solution $u_{N}^{n+1} \in \mathrm{X}_{N}$ on time level $n+1$ is then defined by the equation

$$
\begin{aligned}
\frac{u_{N}^{n+1}\left(x_{j}\right)-u_{N}^{n}\left(x_{j}\right)}{\Delta t}+\mathcal{L}_{\varepsilon} & \left(\frac{u_{N}^{n+1}\left(x_{j}\right)+u_{N}^{n}\left(x_{j}\right)}{2}\right) \\
& =I_{N} \mathcal{N}_{\varepsilon}\left(u_{N}^{n+1}\left(x_{j}\right), u_{N}^{n}\left(x_{j}\right)\right) \quad \forall j \in \mathbb{N}_{N}^{d} .
\end{aligned}
$$

In condensed notation, (3.2) can be restated as follows:

$$
\begin{aligned}
\frac{u_{N}^{n+1}\left(x_{j}\right)-u_{N}^{n}\left(x_{j}\right)}{\Delta t}+\mathcal{L}_{\varepsilon} & u_{N}^{n+1 / 2}\left(x_{j}\right) \\
& =I_{N} \mathcal{N}_{\varepsilon}\left(u_{N}^{n+1}\left(x_{j}\right), u_{N}^{n}\left(x_{j}\right)\right) \quad \forall j \in \mathbb{N}_{N}^{d} .
\end{aligned}
$$


Multiplication of (3.3) by $u_{N}^{n+1}\left(x_{j}\right)-u_{N}^{n}\left(x_{j}\right)$ and summation over the collocation points $x_{j}, j \in \mathbb{N}_{N}^{d}$ using (2.4), yields the following energy equality, which is one of the main features of the scheme.

Lemma 3.1. Consider the sequence $\left(u_{N}^{n}\right)_{n=0}^{J} \subset X_{N}$ with $N \in \mathbb{N}_{>0}$, defined by the Crank-Nicolson scheme (3.2) with initial value $u_{N}^{0}:=I_{N} u_{0} \in X_{N}$, where $u_{0} \in$ $\mathrm{C}\left(\mathbb{T}^{d} ; \mathbb{R}\right)$. Then, letting

$$
E_{N}(v):=\frac{1}{2}\left\langle v, \mathcal{L}_{\varepsilon} v\right\rangle_{N}+\left\langle 1, W_{\varepsilon}(v)\right\rangle_{N}
$$

the following discrete energy law holds:

$$
\frac{1}{\Delta t}\left\|u_{N}^{n+1}-u_{N}^{n}\right\|_{N}^{2}+E_{N}\left(u_{N}^{n+1}\right)=E_{N}\left(u_{N}^{n}\right), \quad n=0,1, \ldots, J-1 .
$$

Next, we consider the existence and uniqueness of solutions to this scheme.

3.2. Existence and uniqueness for the Crank-Nicolson scheme. We shall next show by a fixed-point argument, based on a succession of lemmas proved below, that, for each $n \in\{0,1, \ldots, J-1\}$ and $u_{N}^{n} \in \mathrm{X}_{N}$ given, there exists a unique solution $u_{N}^{n+1} \in \mathrm{X}_{N}$ to (3.2). We begin by restating (3.2) in an equivalent form. By writing $z=u_{N}^{n+1}$ and $v=u_{N}^{n}$, equation (3.2) can be rewritten as follows: given $v \in \mathrm{X}_{N}$, find $z \in \mathrm{X}_{N}$ such that, for all $w \in \mathrm{X}_{N}$,

$$
\langle z, w\rangle_{N}+\frac{\Delta t}{2}\left\langle z, \mathcal{L}_{\varepsilon} w\right\rangle_{N}=\langle v, w\rangle_{N}-\frac{\Delta t}{2}\left\langle v, \mathcal{L}_{\varepsilon} w\right\rangle_{N}+\Delta t\left\langle\mathcal{N}_{\varepsilon}(z, v), w\right\rangle
$$

By defining the adapted inner product $\langle\cdot, \cdot\rangle_{\Delta t}$ and the induced norm $\|\cdot\|_{\Delta t}$ by

$$
\langle v, w\rangle_{\Delta t}:=\langle v, w\rangle_{N}+\frac{\Delta t}{2}\left\langle\mathcal{L}_{\varepsilon} v, w\right\rangle_{N}
$$

and

$$
\|v\|_{\Delta t}^{2}:=\langle v, v\rangle_{\Delta t}=\|v\|_{N}^{2}+\frac{\Delta t}{2}\|v\|_{\mathcal{L}_{\varepsilon}}^{2},
$$

respectively, equation (3.4) can be written as follows:

$$
\langle z, w\rangle_{\Delta t}=\langle v, w\rangle_{\Delta t}+\Delta t\left\langle\mathcal{N}_{\varepsilon}(z, v)-\mathcal{L}_{\varepsilon} v, w\right\rangle_{N} \quad \forall w \in \mathrm{X}_{N} .
$$

Given $v, z \in \mathrm{X}_{N}$, motivated by the form of (3.5) we define $Z=T_{v}^{\varepsilon} z$ as follows:

$$
\langle Z, w\rangle_{\Delta t}=\langle v, w\rangle_{\Delta t}+\Delta t\left\langle\mathcal{N}_{\varepsilon}(z, v)-\mathcal{L}_{\varepsilon} v, w\right\rangle_{N} \quad \forall w \in \mathrm{X}_{N} .
$$

By virtue of the Riesz representation theorem, the operator $T_{v}^{\varepsilon}: \mathrm{X}_{N} \rightarrow \mathrm{X}_{N}$ is well defined. The task of showing the existence of a unique $u_{N}^{n+1} \in \mathrm{X}_{N}$, given $u_{N}^{n} \in \mathrm{X}_{N}$, is then equivalent to showing the existence of a unique fixed point $z$ of $T_{v}^{\varepsilon}$ in $\mathrm{X}_{N}$ with $v:=u_{N}^{n}$ given, and then defining $u_{N}^{n+1}:=z$.

Next, we establish a Lipschitz property of $\mathcal{N}_{\varepsilon}(v, w)$ and in turn the contraction property of $T_{v}^{\varepsilon}$. Suppose for the moment that $\varepsilon=1$. Using definition (3.1) we have, for $v>w$, that

$$
\begin{aligned}
-\frac{\partial \mathcal{N}}{\partial v}(v, w) & =\frac{\mathrm{D} W(v)+\mathcal{N}(v, w)}{v-w} \\
& =\frac{1}{(v-w)^{2}} \int_{w}^{v}(\mathrm{D} W(v)-\mathrm{D} W(s)) \mathrm{d} s \\
& =\frac{1}{(v-w)^{2}} \int_{w}^{v} \int_{s}^{v} \mathrm{D}^{2} W(y) \mathrm{d} y \mathrm{~d} s
\end{aligned}
$$


and similarly for $v<w$. Thus, for all $v, w \in \mathbb{R}, v \neq w$,

$$
\left|\frac{\partial \mathcal{N}}{\partial v}(v, w)\right| \leq \sup \left\{\left|\mathrm{D}^{2} W(y)\right|: y \in[\min (v, w), \max (v, w)]\right\}=: \mathcal{C}(v, w) .
$$

Now, by the mean value theorem,

$$
\left|\mathcal{N}\left(v_{1}, w\right)-\mathcal{N}\left(v_{2}, w\right)\right| \leq \max _{\theta \in[0,1]} \mathcal{C}\left(\theta v_{1}+(1-\theta) v_{2}, w\right)\left|v_{1}-v_{2}\right|
$$

As $L:=\sup \left\{\left|\mathrm{D}^{2} W(y)\right|: y \in \mathbb{R}\right\}$ is finite, we have the uniform Lipschitz property

$$
\left|\mathcal{N}\left(v_{1}, w\right)-\mathcal{N}\left(v_{2}, w\right)\right| \leq L\left|v_{1}-v_{2}\right| \quad \forall v_{1}, v_{2} \in \mathrm{X}_{N}
$$

More generally,

$$
\left|\mathcal{N}_{\varepsilon}\left(v_{1}, w\right)-\mathcal{N}_{\varepsilon}\left(v_{2}, w\right)\right| \leq \frac{L}{\varepsilon}\left|v_{1}-v_{2}\right| \quad \forall v_{1}, v_{2} \in \mathrm{X}_{N} .
$$

By Young's inequality, setting $w=Z-Z^{\prime}$ and subtracting the identities (3.6) for $Z=T_{v}^{\varepsilon} z$ and $Z^{\prime}=T_{v}^{\varepsilon} z^{\prime}$ yields

$$
\left\|Z-Z^{\prime}\right\|_{\Delta t}^{2} \leq(\Delta t)^{2}\left\|\mathcal{N}_{\varepsilon}(z, v)-\mathcal{N}_{\varepsilon}\left(z^{\prime}, v\right)\right\|_{N}^{2}
$$

Thus we have shown the following result.

Lemma 3.2. Let $Z:=T_{v}^{\varepsilon} z$ and $Z^{\prime}:=T_{v}^{\varepsilon} z^{\prime}$, where $v, z, z^{\prime} \in X_{N}$, and suppose that $\Delta t \leq \varepsilon /(2 L)$. Then

$$
\left(\left\|Z-Z^{\prime}\right\|_{N} \leq\right) \quad\left\|Z-Z^{\prime}\right\|_{\Delta t} \leq \frac{1}{2}\left\|z-z^{\prime}\right\|_{N} \quad\left(\leq \frac{1}{2}\left\|z-z^{\prime}\right\|_{\Delta t}\right) .
$$

Consider the sequence $\left(z_{k}\right)_{k=0}^{\infty} \subset \mathrm{X}_{N}$, where $z_{k+1}:=T_{v}^{\varepsilon} z_{k}$ with $z_{0}:=v \in$ $\mathrm{X}_{N}$. By expressing the difference between any two members $z_{\ell}$ and $z_{k+1}$ of the sequence, with $\ell>k+1$, in terms of a telescoping sum of differences of intervening terms, taking norms, using the triangle inequality, the bound above, and summing a geometric progression, we deduce that $\left(z_{k}\right)_{k=1}^{\infty}$ is a Cauchy sequence in the finitedimensional normed linear space $\mathrm{X}_{N}$, with respect to the norm $\|\cdot\|_{\Delta t}$. Hence, by completeness of $\mathrm{X}_{N}$ with respect to the norm $\|\cdot\|_{\Delta t}$, the sequence $\left(z_{k}\right)_{k=0}^{\infty} \subset \mathrm{X}_{N}$, where $z_{k+1}:=T_{v}^{\varepsilon} z_{k}$ with $z_{0}:=v$, converges to a fixed point $z \in \mathrm{X}_{N}$ for any $v \in \mathrm{X}_{N}$. By passing to the limit $\ell \rightarrow \infty$ in the bound on $\left\|z_{\ell}-z_{k+1}\right\|_{\Delta t}$ we deduce that, for $\Delta t \leq \varepsilon /(2 L)$

$$
\left\|z-z_{k+1}\right\|_{\Delta t} \leq 2^{-k}\left\|z_{1}-v\right\|_{\Delta t} .
$$

It remains to control the size of $\left\|z_{1}-v\right\|_{\Delta t}$, where $v$ is the initial iterate and $z_{1}$ is the first iterate in the iteration scheme. To do so, let $w:=z_{1}-v=T_{v}^{\varepsilon} v-v$. Then

$$
\begin{aligned}
\|w\|_{\Delta t}^{2} & =\Delta t\left\langle\mathcal{N}_{\varepsilon}(v)-\mathcal{L}_{\varepsilon} v, w\right\rangle_{N} \\
& \leq \frac{1}{2}(\Delta t)^{2}\left\|\mathrm{D} W_{\varepsilon}(v)\right\|_{N}^{2}+\frac{1}{2}\|w\|_{N}^{2}+\Delta t\left\langle\mathcal{L}_{\varepsilon} v, v\right\rangle_{N}+\frac{\Delta t}{4}\left\langle\mathcal{L}_{\varepsilon} w, w\right\rangle_{N},
\end{aligned}
$$

where we used that $\mathcal{N}_{\varepsilon}(v)=\mathcal{N}_{\varepsilon}(v, v)=-\mathrm{D} W_{\varepsilon}(v)$. By transferring the terms involving $w$ from the right-hand side to the left-hand side and multiplying the resulting inequality by 2 , we obtain the following result.

Lemma 3.3. Suppose that $\Delta t \leq \varepsilon /(2 L)$. Then

$$
\left\|T_{v}^{\varepsilon} v-v\right\|_{\Delta t}^{2} \leq(\Delta t)^{2}\left\|D W_{\varepsilon}(v)\right\|_{N}^{2}+2 \Delta t\left\langle\mathcal{L}_{\varepsilon} v, v\right\rangle_{N} \quad \forall v \in X_{N} .
$$


With the bound stated in Lemma 3.3 we now return to the analysis of the fixedpoint iteration operator $T_{v}^{\varepsilon}: \mathrm{X}_{N} \rightarrow \mathrm{X}_{N}$ associated with the problem

$$
\langle Z, w\rangle_{\Delta t}=\langle v, w\rangle_{\Delta t}+\Delta t\left\langle\mathcal{N}_{\varepsilon}(z, v)-\mathcal{L}_{\varepsilon} v, w\right\rangle_{N},
$$

where $\mathcal{N}_{\varepsilon}(z, v)$ is defined as in (3.1) by

$$
W_{\varepsilon}(\cdot):=\frac{1}{\varepsilon} W(\cdot) \quad \text { and } \quad \mathcal{L}_{\varepsilon}:=\varepsilon(-\Delta)+\sigma(\mathrm{D}) .
$$

Note that the inner product $\langle\cdot, \cdot\rangle_{\Delta t}=\langle\cdot, \cdot\rangle_{\Delta t, \varepsilon}$ depends on $\varepsilon$ too, but we will suppress this dependence in our notation. As discussed in the Introduction, we assume that $W$ is a nonnegative double-well potential with wells at \pm 1 and quadratic growth at infinity in the sense that $\left|\mathrm{D}^{2} W(v)\right| \leq L$ and $|\mathrm{D} W(v)|^{2} \leq K W(v)$ for all $v \in \mathbb{R}$; cf. (1.3). A typical example of such a double-well potential is a truncated Ginzburg-Landau potential

$$
W(v)=c_{0} \frac{\left(1-v^{2}\right)^{2}}{1+v^{2}}
$$

with a renormalization constant $c_{0}>0$ chosen so that $\int_{-1}^{1} \sqrt{2 W(v)} \mathrm{d} v=1$ and

$$
-\mathrm{D} W(v)=c_{0} v \frac{1-v^{2}}{1+v^{2}}\left(4+2 \frac{1-v^{2}}{1+v^{2}}\right) .
$$

We shall now rephrase the bound from Lemma 3.3 in terms of $\varepsilon$ and the associated energy

$$
E_{N}(v):=\frac{1}{2}\left\langle\mathcal{L}_{\varepsilon} v, v\right\rangle_{N}+\left\langle 1, W_{\varepsilon}(v)\right\rangle_{N} .
$$

Note that if $\Delta t \leq \varepsilon /(2 L)$, then, by Lemma 3.3 and for $M_{0}:=\max \left\{\frac{K}{2 L}, 4\right\}$, we have

$$
\left\|T_{v}^{\varepsilon} v-v\right\|_{\Delta t}^{2} \leq M_{0} \Delta t E_{N}(v) \text {. }
$$

Hence we obtain from (3.8) and (3.9) the following result.

Theorem 3.4. Suppose that $W \in C^{2}(\mathbb{R})$ is nonnegative and there exist positive real numbers $K$ and $L$ such that

$$
|D W(v)|^{2} \leq K W(v) \quad \text { and } \quad\left|D^{2} W(v)\right| \leq L \quad \forall v \in \mathbb{R} .
$$

Suppose further that, for $v \in X_{N}, \varepsilon>0$ fixed and $N \geq 1$,

$$
E_{N}(v)<\infty \text { and } \Delta t \leq \varepsilon /(2 L) .
$$

Then, the sequence $\left(z_{k}\right)_{k=0}^{\infty}$, generated by the fixed-point iteration $z_{k+1}:=T_{v}^{\varepsilon} z_{k}$ with initial value $z_{0}:=v \in X_{N}$, converges in $\mathrm{L}^{2}\left(\mathbb{T}^{d} ; \mathbb{C}\right)$ to a fixed point $z \in X_{N}$, with $E_{N}(z) \leq E_{N}(v)$ such that

$$
\left\|z-z_{k+1}\right\|_{\Delta t}^{2} \leq 4^{-k} M_{0} \Delta t E_{N}(v) \quad \text { for any } k \geq 0,
$$

where $M_{0}:=\max \left\{\frac{K}{2 L}, 4\right\}$. In particular, $M_{0}$ is independent of $N, \Delta t, \varepsilon$ and the dimension $d$, although the range of $\Delta t$ for which the result holds is dependent on $\varepsilon / L$ (but not on $K$ or $N$ ).

Denoting by $u_{N}^{n+1}$ the unique fixed point $z$ in $\mathrm{X}_{N}$ of $T_{v}^{\varepsilon}$ with $v=u_{N}^{n} \in \mathrm{X}_{N}$, for $\Delta t \leq \varepsilon /(2 L)$ and $N \geq 1$, Theorem 3.4 implies the existence of a unique solution to the $(n+1)$ st step of the Crank-Nicolson scheme (3.2). Proceeding inductively for $n=0,1, \ldots, J-1$, we then deduce the existence of a unique solution $\left(u_{N}^{n}\right)_{n=0}^{J} \subset \mathrm{X}_{N}$ to the scheme for a given initial value $u_{N}^{0}=I_{N} u_{0} \in \mathrm{X}_{N}$, for all $\Delta t \leq \varepsilon /(2 L)$ and all $N \geq 1$. 


\section{A PRIORI ERROR ESTIMATES}

In this section we keep the parameter $\varepsilon$ fixed (and therefore, for the sake of clarity of exposition, omit the subscript $\varepsilon$ from $\mathcal{N}_{\varepsilon}$ and $W_{\varepsilon}$ ) and we assume in addition that $W$ has a uniformly bounded third derivative on $\mathbb{R}$. The argument below is inspired by the one in 13 . We let $u$ be the solution of the exact equation $u_{t}+\mathcal{L} u=\mathcal{N}(u)$. Letting $e_{N}^{n}=u_{N}^{n}-P_{N} u\left(t^{n}\right)$, where $P_{N}$ is the $\mathrm{L}^{2}\left(\mathbb{T}^{d} ; \mathbb{C}\right)$-orthogonal projection operator, and defining

$$
e_{N}^{n+1 / 2}:=\frac{1}{2}\left(e_{N}^{n}+e_{N}^{n+1}\right)
$$

we obtain from the discrete and continuous equation with $e_{N}^{n+1 / 2}$ as a test function and the Cauchy-Schwarz inequality,

$$
\begin{aligned}
& \frac{1}{2 \Delta t}\left(\left\|e_{N}^{n+1}\right\|^{2}-\left\|e_{N}^{n}\right\|^{2}\right)+\left\|e_{N}^{n+1 / 2}\right\|_{\mathcal{L}}^{2} \\
& \quad \leq\left(\left\|\mathcal{N}\left(u_{N}^{n}, u_{N}^{n+1}\right)-\mathcal{N}\left(u\left(t^{n+1 / 2}\right)\right)\right\|_{N}+\left\|\frac{u\left(t^{n+1}\right)-u\left(t^{n}\right)}{\Delta t}-u_{t}\left(t^{n+1 / 2}\right)\right\|\right. \\
& \left.\quad+\left\|R_{N} \mathcal{N}\left(u\left(t^{n+1 / 2}\right)\right)\right\|\right)\left\|e_{N}^{n+1 / 2}\right\|+\left\|\frac{u\left(t^{n}\right)+u\left(t^{n+1}\right)}{2}-u\left(t^{n+1 / 2}\right)\right\|_{\mathcal{L}}\left\|e_{N}^{n+1 / 2}\right\|_{\mathcal{L}},
\end{aligned}
$$

with the energy norm $\|v\|_{\mathcal{L}}^{2}:=(\mathcal{L} v, v)_{\mathrm{L}^{2}\left(\mathbb{T}^{d} ; \mathbb{C}\right)}$ and where $R_{N}:=P_{N}-I_{N}$. Therefore, by Young's inequality, we have

$$
\begin{aligned}
\frac{1}{\Delta t}( & \left.\left\|e_{N}^{n+1}\right\|^{2}-\left\|e_{N}^{n}\right\|^{2}\right)+\left\|e_{N}^{n+1 / 2}\right\|_{\mathcal{L}}^{2} \leq 3\left\|e_{N}^{n+1 / 2}\right\|^{2} \\
& +\left(\left\|\mathcal{N}\left(u_{N}^{n}, u_{N}^{n+1}\right)-\mathcal{N}\left(u\left(t^{n+1 / 2}\right)\right)\right\|_{N}^{2}+\left\|R_{N} \mathcal{N}\left(u\left(t^{n+1 / 2}\right)\right)\right\|^{2}\right) \\
& +\left\|\frac{u\left(t^{n}\right)+u\left(t^{n+1}\right)}{2}-u\left(t^{n+1 / 2}\right)\right\|_{\mathcal{L}}^{2}+\left\|\frac{u\left(t^{n+1}\right)-u\left(t^{n}\right)}{\Delta t}-u_{t}\left(t^{n+1 / 2}\right)\right\|^{2}
\end{aligned}
$$

By virtue of Taylor's theorem the last two terms can be bounded by

$$
c(\Delta t)^{3} \int_{t^{n}}^{t^{n+1}}\left(\left\|u_{t t}(t)\right\|_{\mathcal{L}}^{2}+\left\|u_{t t t}(t)\right\|^{2}\right) \mathrm{d} t
$$

By the definition of $\mathcal{N}(v, w)$ and with an interpretation as a central difference quotient we have

$$
\left|\mathcal{N}(v, w)-\mathcal{N}\left(\frac{v+w}{2}\right)\right| \leq C|v-w|^{2}
$$

where, by Taylor's theorem, $C=\frac{1}{24} \sup _{y \in[\min (v, w), \max (v, w)]}\left|\mathrm{D}^{2} \mathcal{N}(y)\right|$. By the Lipschitz property (3.7), the inequality

$$
\left|\mathcal{N}\left(u_{1}, w\right)-\mathcal{N}\left(u_{2}, w\right)\right| \leq L\left|u_{1}-u_{2}\right|
$$

holds, with $L$ independent of $w \in \mathbb{R}$. We obtain the following lemma, which is an adaptation (and, in fact, a generalization) of Lemma 4.3 in [6].

Lemma 4.1. Suppose that $\mathcal{N}(v)=-D W(v)$ satisfies a uniform Lipschitz condition and has a bounded second derivative; then, there exists a constant $c>0$ that only 
depends on $\mathcal{N}$ such that

$$
\begin{aligned}
\| \mathcal{N}\left(u_{N}^{n}, u_{N}^{n+1}\right) & -\mathcal{N}\left(u\left(t^{n+1 / 2}\right)\right) \|_{N}^{2} \leq c\left(\left\|u_{N}^{n}-u\left(t^{n}\right)\right\|_{N}^{2}+\left\|u_{N}^{n+1}-u\left(t^{n+1}\right)\right\|_{N}^{2}\right. \\
& \left.+\left\|\left(u\left(t^{n+1}\right)-u\left(t^{n}\right)\right)^{2}\right\|_{N}^{2}+\left\|\frac{u\left(t^{n+1}\right)+u\left(t^{n}\right)}{2}-u\left(t^{n+1 / 2}\right)\right\|_{N}^{2}\right) .
\end{aligned}
$$

By virtue of Taylor's theorem the bound provided in the lemma can be estimated further:

$$
\begin{aligned}
& \left\|\mathcal{N}\left(u_{N}^{n}, u_{N}^{n+1}\right)-\mathcal{N}\left(u\left(t^{n+1 / 2}\right)\right)\right\|_{N}^{2} \leq c\left(\left\|e_{N}^{n}\right\|_{N}^{2}+\left\|e_{N}^{n+1}\right\|_{N}^{2}\right. \\
& \left.+\left\|R_{N} u\left(t^{n}\right)\right\|^{2}+\left\|R_{N} u\left(t^{n+1}\right)\right\|^{2}+(\Delta t)^{3} \int_{t^{n}}^{t^{n+1}}\left\|u_{t t}(t)\right\|_{N}^{2}+\left\|\left(u_{t}(t)\right)^{2}\right\|_{N}^{2} \mathrm{~d} t\right)
\end{aligned}
$$

where $R_{N} u$ is the aliasing error. Finally, we obtain from (4.1), (4.2) and (4.3):

$$
\begin{aligned}
\left\|e_{N}^{n+1}\right\|^{2}-\left\|e_{N}^{n}\right\|^{2}+\Delta t\left\|e_{N}^{n+1 / 2}\right\|_{\mathcal{L}}^{2} \\
\leq c(\Delta t)^{4} \int_{t^{n}}^{t^{n+1}}\left(\left\|u_{t t t}(t)\right\|^{2}+\left\|u_{t t}(t)\right\|_{\mathcal{L}}^{2}+\left(\left\|u_{t t}(t)\right\|_{N}^{2}+\left\|\left(u_{t}(t)\right)^{2}\right\|_{N}^{2}\right)\right) \mathrm{d} t \\
\quad+c \Delta t\left(\left\|e_{N}^{n}\right\|^{2}+\left\|e_{N}^{n+1 / 2}\right\|^{2}+\left\|e_{N}^{n+1}\right\|^{2}\right. \\
\left.\quad+\left\|R_{N} u\left(t^{n}\right)\right\|^{2}+\left\|R_{N} u\left(t^{n+1}\right)\right\|^{2}+\left\|R_{N} \mathcal{N}\left(u\left(t^{n+1 / 2}\right)\right)\right\|^{2}\right),
\end{aligned}
$$

and after summation from $n=0$ to $k-1$, where $1 \leq k \leq J$,

$$
\begin{aligned}
& \left\|e_{N}^{k}\right\|^{2}+\Delta t \sum_{n=0}^{k-1}\left\|e_{N}^{n+1 / 2}\right\|_{\mathcal{L}}^{2} \\
& \leq c(\Delta t)^{4} \int_{0}^{T}\left(\left\|u_{t t t}(t)\right\|^{2}+\left\|u_{t t}(t)\right\|_{\mathcal{L}}^{2}+\left(\left\|u_{t t}(t)\right\|_{N}^{2}+\left\|\left(u_{t}(t)\right)^{2}\right\|_{N}^{2}\right)\right) \mathrm{d} t \\
& \quad+c \Delta t \sum_{n=0}^{k}\left(\left\|e_{N}^{n}\right\|^{2}+\left\|R_{N} u\left(t^{n}\right)\right\|^{2}+\left\|R_{N} \mathcal{N}\left(u\left(t^{n+1 / 2}\right)\right)\right\|^{2}\right) .
\end{aligned}
$$

Regarding the third and fourth term under the integral sign, we recall that in view of the Sobolev embedding $\|v\|_{N} \lesssim\|v\|_{\mathrm{L}^{\infty}\left(\mathbb{T}^{d} ; \mathbb{C}\right)} \lesssim\|v\|_{s}$ for $s>d / 2$. Since $\mathrm{H}^{s}\left(\mathbb{T}^{d} ; \mathbb{C}\right)$ is a Banach algebra for $s>d / 2$, we also have that $\left\|v^{2}\right\|_{s} \lesssim\|v\|_{s}^{2}$. Therefore, in the case of $d=2,3$ the integral terms are finite, provided

$$
u_{t} \in \mathrm{H}_{t}^{2} \mathrm{~L}_{x}^{2} \cap \mathrm{H}_{t}^{1} \mathrm{H}_{x}^{2}\left((0, T) \times \mathbb{T}^{d}\right) .
$$

Turning to the third line of (4.4), according to (2.3) we have, for $d=2,3$,

$$
\left\|R_{N} u(t)\right\| \lesssim N^{-2}\|u(t)\|_{2},
$$

and similarly, if $W$ is three times differentiable on $\mathbb{R}$ and $v \in \mathbb{R} \mapsto|\mathrm{D} \mathcal{N}(v)|=$ $\left|\mathrm{D}^{2} W(v)\right|$ and $v \in \mathbb{R} \mapsto\left|\mathrm{D}^{2} \mathcal{N}(v)\right|=\left|\mathrm{D}^{3} W(v)\right|$ are bounded functions, we have that

$$
\left\|R_{N} \mathcal{N}(u(t))\right\| \lesssim N^{-2}\|\mathcal{N}(u(t))\|_{2} \lesssim N^{-2}\|u(t)\|_{2}, \quad t \geq 0
$$


Hence the terms containing $R_{N}$ can be bounded provided $\mathrm{L}_{t}^{\infty} \mathrm{H}_{x}^{2}\left((0, T) \times \mathbb{T}^{d}\right)$. More generally, if $W$ is $\ell+1$ times differentiable on $\mathbb{R}, \ell \geq 2$, so that

$$
\sup _{v \in \mathbb{R}}\left(\left|\mathrm{D}^{2} W(v)\right|+\cdots+\left|\mathrm{D}^{\ell+1} W(v)\right|\right)<\infty
$$

(cf. (1.3) and (1.4) ) and $u \in \mathrm{H}_{t}^{3} \mathrm{~L}_{x}^{2} \cap \mathrm{H}_{t}^{2} \mathrm{H}_{x}^{s} \cap \mathrm{L}_{t}^{\infty} \mathrm{H}_{x}^{s}\left((0, T) \times \mathbb{T}^{d}\right)$ for $2 \leq s \leq \ell$, then

$$
\left\|R_{N} u(t)\right\|+\left\|R_{N} \mathcal{N}(u(t))\right\| \lesssim N^{-s}\|u(t)\|_{s}, \quad t \geq 0 .
$$

We can then apply the discrete Gronwall inequality to (4.4) and obtain the following result.

Theorem 4.2. Suppose that $d=2,3, W$ is $\ell+1$ times differentiable on $\mathbb{R}$ for some $\ell \geq 2$ and satisfies (1.3) and (1.4), and that for some $s, 2 \leq s \leq \ell$, we have

$$
u \in \mathrm{H}_{t}^{3} \mathrm{~L}_{x}^{2} \cap \mathrm{H}_{t}^{2} \mathrm{H}_{x}^{2} \cap \mathrm{L}_{t}^{\infty} \mathrm{H}_{x}^{s}\left((0, T) \times \mathbb{T}^{d}\right) .
$$

Then, there exists a positive constant $C=C(T / \varepsilon)$ such that

$$
\max _{1 \leq n \leq J}\left\|u_{N}^{n}-u\left(t^{n}\right)\right\| \leq C\left(N^{-s}+(\Delta t)^{2}\right) .
$$

Note that by virtue of Sobolev embedding the assumption $u \in \mathrm{L}_{t}^{\infty} \mathrm{H}_{x}^{s}\left((0, T) \times \mathbb{T}^{d}\right)$ can be omitted in the case of $s=2$.

The next section is devoted to numerical experiments aimed at assessing the practical performance of the proposed numerical method and to undertaking a parameter study of the energy functional (1.1).

\section{NumericAl EXPERIMENTS}

We have implemented the numerical scheme (3.2) in MATLAB. Our numerical experiments focus on the two-dimensional domain $\mathbb{T}^{2}=(0,2 \pi)^{2}$. For the purpose of our parameter studies we reintroduce the parameter $\gamma>0$, the interfacial energy per unit length, which balances the relative strength of the domain-wall energy against the dipolar energy. We consider, for $\varepsilon>0$, the linear and nonlinear operators

$$
v \mapsto \mathcal{L}_{\varepsilon} v=\gamma \varepsilon(-\Delta) v+\sigma(\mathrm{D}) v
$$

and

$$
v \mapsto \mathcal{N}_{\varepsilon}(v)=-\frac{\gamma}{\varepsilon} \mathrm{D} W(v),
$$

where $\sigma: k \in \mathbb{Z}^{d} \mapsto \sigma(k) \in \mathbb{R}_{\geq 0}$ is a Fourier multiplier

$$
\sigma(k)=\frac{1-\exp (-|k|)}{|k|} \text { for } k \neq 0 \text { and } \sigma(0)=1
$$

and $W: v \in \mathbb{R} \mapsto W(v) \in \mathbb{R}_{\geq 0}$ is the double-well potential

$$
W(v)=c_{0} \frac{\left(1-v^{2}\right)^{2}}{1+v^{2}} \quad \text { where } \quad c_{0}=\frac{1}{2}\left(\int_{-1}^{1} \frac{1-v^{2}}{\sqrt{1+v^{2}}} \mathrm{~d} v\right)^{-2} .
$$

Our model problem is motivated by micromagnetic models for garnet films, where $\sigma$ appears in reduced representations for the dipolar energy; cf. [9]. As indicated in the Introduction such models include an additional dilation parameter $\delta>0$, which corresponds to the relative film thickness. The precise energy scaling and morphology, depending on all system parameters, is subtle and is hard to capture in a rigorous analytic fashion. Current investigations reported in the physics literature mainly rely on further reductions and optimization through a special ansatz; cf. 
7. 10. Our numerical experiments provide an adequate account of characteristic patterns and structures in more generality and support the latter results.

5.1. Procedure. The implementation is based on the nonlinear system

$$
\begin{array}{r}
u_{N}^{n+1}\left(x_{j}\right)=\left(1+\frac{\Delta t}{2} \mathcal{L}_{\varepsilon}\right)^{-1}\left[\left(1-\frac{\Delta t}{2} \mathcal{L}_{\varepsilon}\right) u_{N}^{n}\left(x_{j}\right)+\Delta t I_{N} \mathcal{N}_{\varepsilon}\left(u_{N}^{n+1}\left(x_{j}\right), u_{N}^{n}\left(x_{j}\right)\right)\right], \\
j \in \mathbb{N}_{N}^{2}=\{0,1,2, \ldots, N-1\}^{2},
\end{array}
$$

that we solve at each time-step by a fixed-point iteration. Inspection in Fourier space shows that the inverse $\left(1+\frac{\Delta t}{2} \mathcal{L}_{\varepsilon}\right)^{-1}$ is well defined. In order to compute the numerical solution at time iteration $n+1$, we use the following predictor-corrector algorithm:

$$
\begin{array}{r}
u_{N,[0]}^{n+1}\left(x_{j}\right)=\left(1+\frac{\Delta t}{2} \mathcal{L}_{\varepsilon}\right)^{-1}\left[\left(1-\frac{\Delta t}{2} \mathcal{L}_{\varepsilon}\right) u_{N}^{n}\left(x_{j}\right)+\Delta t I_{N} \mathcal{N}_{\varepsilon}\left(u_{N}^{n}\left(x_{j}\right), u_{N}^{n}\left(x_{j}\right)\right)\right], \\
j \in \mathbb{N}_{N}^{2}=\{0,1,2, \ldots, N-1\}^{2}, \\
u_{N,[k+1]}^{n+1}\left(x_{j}\right)=\left(1+\frac{\Delta t}{2} \mathcal{L}_{\varepsilon}\right)^{-1}\left[\left(1-\frac{\Delta t}{2} \mathcal{L}_{\varepsilon}\right) u_{N}^{n}\left(x_{j}\right)+\Delta t I_{N} \mathcal{N}_{\varepsilon}\left(u_{N,[k]}^{n}\left(x_{j}\right), u_{N}^{n}\left(x_{j}\right)\right)\right], \\
j \in \mathbb{N}_{N}^{2}=\{0,1,2, \ldots, N-1\}^{2}, \quad k=0,1,2, \ldots .
\end{array}
$$

The iterations to solve the system at each time-step are considered to have converged when the discrete $\mathrm{L}^{2}(\Omega)$ norm of the difference of two successive iterates becomes smaller than a chosen positive tolerance; in our case the stopping tolerance was set to $10^{-8}$. The quantities on the right-hand side of the above equations are computed by applying the following procedure:

1. Evaluation of $\left(1-\frac{\Delta t}{2} \mathcal{L}_{\varepsilon}\right) u_{N}^{n}\left(x_{j}\right)$ in Fourier space;

2. Evaluation of the quantity $\Delta t I_{N} \mathcal{N}_{\varepsilon}\left(u_{N,[k]}^{n}\left(x_{j}\right), u_{N}^{n}\left(x_{j}\right)\right)$ in physical space, followed by switching to Fourier space using Fast Fourier Transform (FFT);

3. Evaluation of $\left(1+\frac{\Delta t}{2} \mathcal{L}_{\varepsilon}\right)^{-1}\left[\left(1-\frac{\Delta t}{2} \mathcal{L}_{\varepsilon}\right) u_{N}^{n}\left(x_{j}\right)+\Delta t I_{N} \mathcal{N}_{\varepsilon}\left(u_{N,[k]}^{n}\left(x_{j}\right), u_{N}^{n}\left(x_{j}\right)\right)\right]$ in Fourier space, followed by an Inverse Fast Fourier Transform (IFFT) to return to physical space.

The terminal time $T=J \Delta t$ for the evolution, i.e., the total number of time-steps $J$, is determined through a smallness condition for the energy gradient, which indicates that a local minimum has been reached approximately.

Remark 5.1. Our numerical analysis shows the convergence of the numerical scheme for temporal meshes with uniform spacing $\Delta t$, once $\Delta t$ is sufficiently small relative to $\varepsilon$. However, with regard to the different time-scales involved in the morphological evolution of the patterns (quick formation of domains at the beginning, much slower evolution afterwards), adaptive time-stepping based on the number of iterations needed to solve the fixed-point iteration was also implemented in the algorithm. This allows us to locally (in time) adapt the time-step to the evolution of the solution: on time intervals of slow/fast evolution the time-step is increased/decreased, respectively. This simple step-size selection process reduces drastically the computing time needed to reach a local minimum of the free energy.

5.2. Morphological evolution and interpretation. Figures [1] (a) (e) and Figures 2(a) (e) show typical examples of temporal evolution of morphological patterns during spinodal decomposition and subsequent growth. Black represents positive 


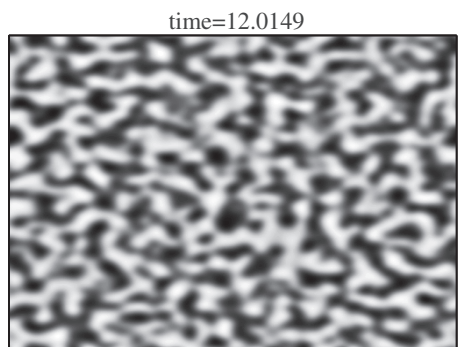

(a)

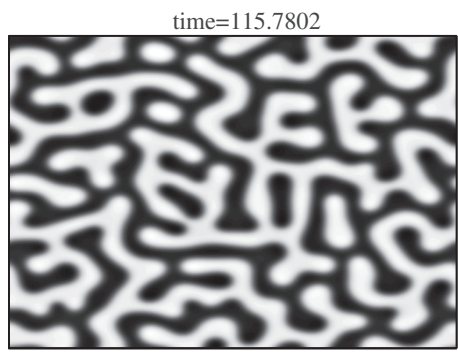

(c)

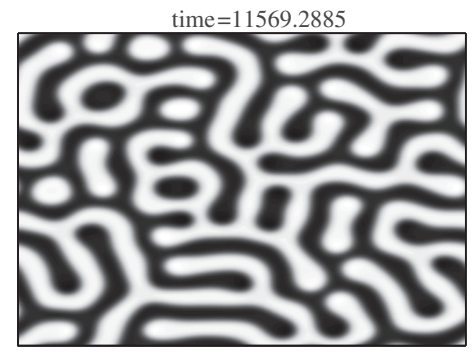

(e)

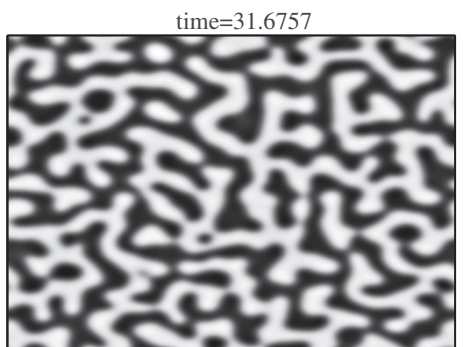

(b)

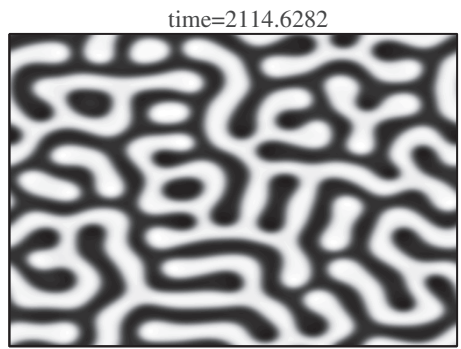

(d)

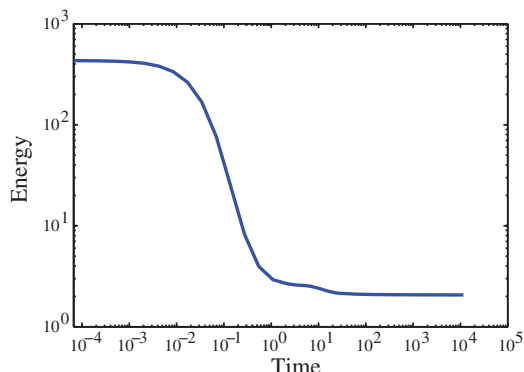

(f)

Figure 1. (a) (e) Formation and temporal evolution of a labyrinth pattern obtained for a randomly distributed initial condition. (f) Temporal evolution of the associated discrete energy.

values, typically around +1 , and white represents negative values around -1 . During the spinodal decomposition, we observe the formation of domains on a relatively short time-scale and with a corresponding substantial drop in the value of the discretized free energy of the system. During the succeeding growth period, we observe domain expansion and possibly nucleation on a much longer time-scale. The decrease of the energy on that period is also much smaller.

Figures [1] (a) (e) show the formation and evolution of a so-called labyrinth pattern that typically arises in the study of magnetic garnet films. It was obtained for randomly distributed initial values on a lattice of $512 \times 512$ grid points and for parameter values $\gamma=1 / 100$ and $\varepsilon=1 / 20$. 
Figures 2(a) (e) show the evolution obtained for the initial condition $u_{0}$ defined by

$$
u_{0}\left(x_{1}, x_{2}\right):=\sin \left(4 x_{1}\right) \sin \left(4 x_{2}\right)
$$

discretized on a lattice of $512 \times 512$ grid points and parameter values $\gamma=1 / 100$ and $\varepsilon=1 / 20$. The drops of the energy seen in Figure 2/(f) each correspond to topological changes in the pattern: the first drop occurs during the transition from the initial state to the checkerboard pattern, the second one during the transition from the checkerboard pattern to the stripe pattern. The last decrease, which is much less prominent, occurs during the transition from the rectilinear pattern to the curly-stripe pattern.

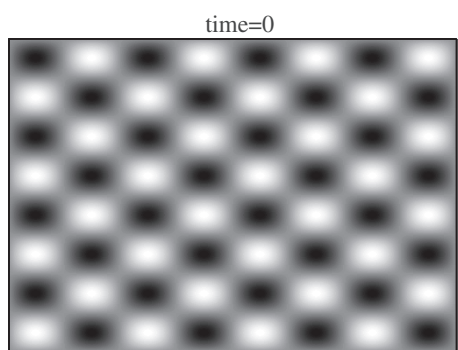

(a)

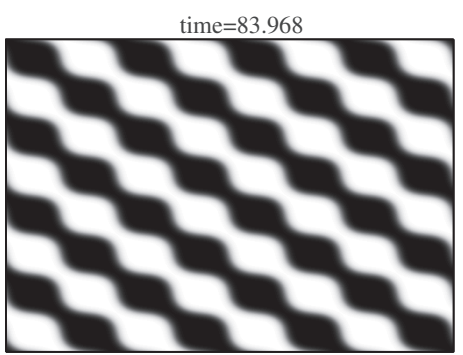

(c)

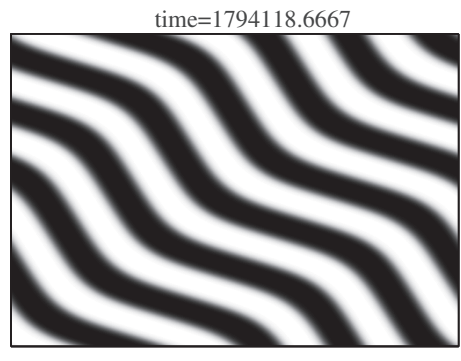

(e)

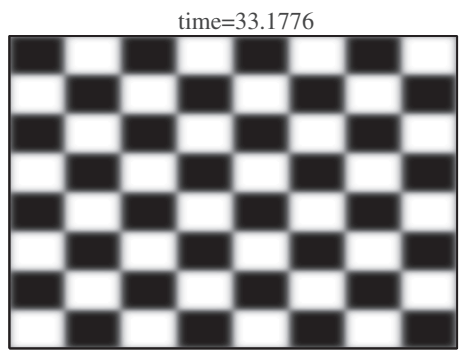

(b)

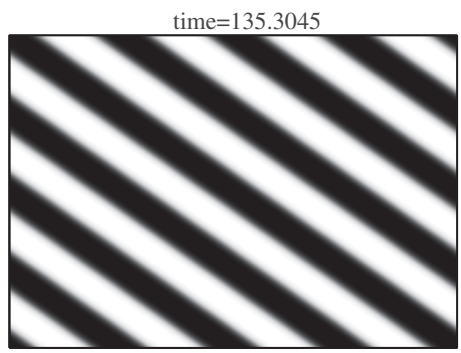

(d)

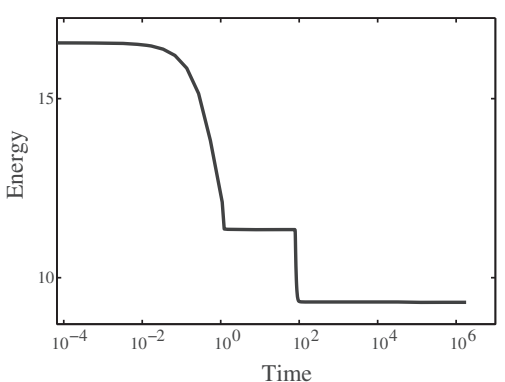

(f)

FiguRE 2. (a) (e) Formation of a checkerboard pattern and subsequent evolution towards a stripe pattern. (f) The corresponding energy profile. 


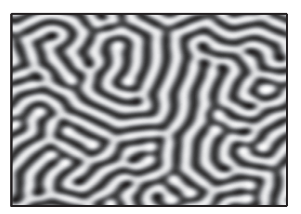

(a)

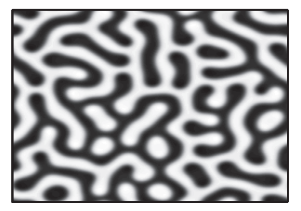

(b)

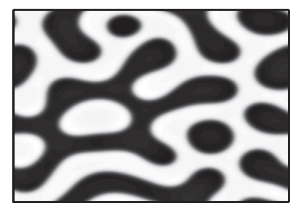

(c)

FiguRE 3. Typical domain sizes observed for increasing values of the interfacial energy parameter, respectively, (a) $\gamma=1 / 300$, (b) $\gamma=1 / 100$ and $(\mathrm{c}) \gamma=1 / 20$.

Based on a reduction of (1.1) through corresponding trial functions and an optimization argument, Gehring and Kaplan [7] showed that a stripe pattern is a lower energy state than a checkerboard pattern. The numerical results shown in Figure 2 are consistent with these predictions. Indeed, the checkerboard pattern obtained in Figure 2(b) appears as an unstable configuration, as we observe a further evolution of the system into an energetically lower, stripe pattern, configuration; cf. Figure 2](d)]

5.3. Parameter-dependence. We have investigated the influence of the interfacial energy parameter $\gamma$ on the domain morphology and the scaling of the asymptotic energy. Qualitatively, a decrease in the typical domain wall energy $\gamma$ triggers the influence of the dipolar interaction, and therefore tends to favor oscillating solutions and eventually microstructure. Consequently, as displayed in Figure 3, we observe a decrease of the typical size of the domains. In the opposite regime, for increasing values of the interfacial energy parameter, the dipolar interaction has a declining influence. For sufficiently large $\gamma$, the problem almost reduces to the minimal interface problem. In that case, the absolute minimizer is a single domain state taking the value either +1 or -1 everywhere. This statement is illustrated in Figure 4. The pattern-evolution was obtained again for the initial condition (5.1), but with the parameter value $\gamma=1000$.

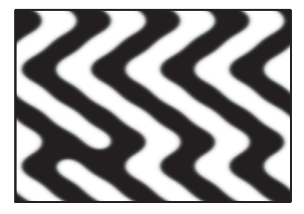

(a)

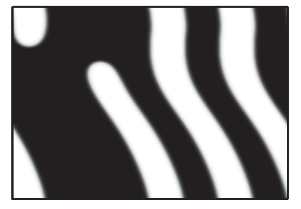

(b)

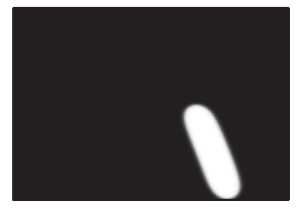

(c)

Figure 4. Temporal evolution towards a single-domain state. The patterns were obtained on a $512 \times 512$ grid for the initial condition $u_{0}\left(x_{1}, x_{2}\right)=\sin \left(4 x_{1}\right) \sin \left(4 x_{2}\right)$ and the parameter value $\gamma=1000$.

In a more quantitative study we have investigated the precise scaling law for the energy as $\gamma$ varies. As pointed out earlier, real micromagnetic applications involve a second parameter $\delta$ that corresponds to the film thickness relative to the dimension of the periodicity cell. Formal results have been obtained in the physics literature: the theory of Kooy and Enz 10, predicts an algebraic energy scaling of 
order $(\gamma / \delta)^{1 / 2}$ for $\gamma \ll \delta$, whereas for $\gamma \gg \delta$ the approach of Gehring and Kaplan 7] predicts an exponential dependence of type $\delta C_{1}\left(1-C_{2} \exp \left[-C_{3}(\gamma / \delta)\right]\right)$ with positive constants $C_{1}, C_{2}$ and $C_{3}$.

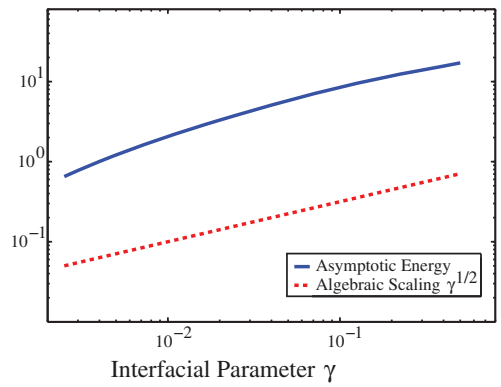

(a)

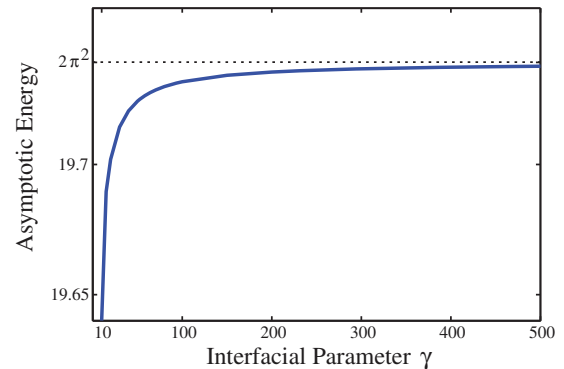

(b)

FiguRE 5. Evolution of the asymptotic discrete energy as a function of the interfacial energy parameter $\gamma$. (a) Logarithmic plot for values of $\gamma$ ranging from $1 / 400$ to $1 / 2$ and comparison with the algebraic scaling $\gamma^{1 / 2}$ predicted by [10]. (b)]Plot of the asymptotic discrete energy for values of $\gamma$ ranging from 10 to 500 .

In our model problem we bypass the additional complexity arising from the presence of a thickness parameter $\delta$ by setting it to 1 for convenience. Accordingly, we have studied the evolution of the asymptotic energy as a function of the interfacial energy parameter $\gamma$ only. Taking a random initial condition and $\varepsilon=1 / 20$, we ran the algorithm for values of $\gamma$ ranging from $1 / 400$ to $1 / 2$ in the first instance, and subsequently for values ranging from 10 to 500 . For the small values of the interfacial energy parameter, we obtain a scaling of the asymptotic energy as an algebraic power of $\gamma$; cf. Figure [a $[$ (a), For larger values of $\gamma$, the solutions converge towards a single domain state. The stepwise decrease in the energy is much less prominent than in the previous case; nevertheless, the plot of the asymptotic energy as a function of $\gamma$ suggests a scaling law of exponential type, as predicted by Kaplan and Gehring; cf. Figure [5] (b). These scaling laws are consistent with the formal results predicted in [10] and 7], and a crossover in the energy scaling can be observed.

\section{ACKNOWLEDGMENT}

We thank the anonymous Referees for helpful suggestions.

\section{REFERENCES}

1. G. Alberti, Variational models for phase transitions, an approach via $\Gamma$-convergence, Calculus of variations and partial differential equations (Pisa, 1996), Springer, Berlin, 2000, pp. 95-114. MR. 1757697

2. A. Braides, $\Gamma$-convergence for beginners, Oxford Lecture Series in Mathematics and its Applications, vol. 22, Oxford University Press, Oxford, 2002. MR1968440 (2004e:49001)

3. C. Canuto and A. Quarteroni, Approximation results for orthogonal polynomials in Sobolev spaces, Math. Comp. 38 (1982), no. 157, 67-86. MR637287 (82m:41003)

4. R. Choksi, Scaling laws in microphase separation of diblock copolymers, J. Nonlinear Sci. 11 (2001), no. 3, 223-236. MR1852942 (2003h:82091) 
5. G. Dal Maso, An introduction to $\Gamma$-convergence, Progress in Nonlinear Differential Equations and their Applications, 8, Birkhäuser Boston, Inc., Boston, MA, 1993. MR.1201152 (94a:49001)

6. Q. Du and R. A. Nicolaides, Numerical analysis of a continuum model of phase transition, SIAM J. Numer. Anal. 28 (1991), no. 5, 1310-1322. MR.1119272 (92h:65166)

7. G.A. Gehring and B. Kaplan, The domain structure in ultrathin magnetic films, J. Magn. Mat. 128 (1993), 111-116.

8. D. Henry, Geometric Theory of Semilinear Parabolic Equations, Lecture Notes in Mathematics, vol. 840, Springer-Verlag, Berlin, 1981. MR610244 (83j:35084)

9. A. Hubert and R. Schäfer, Magnetic Domains: The Analysis of Magnetic Microstructures, Springer-Verlag, Berlin-Heidelberg-New York, 1998.

10. C. Kooy and U. Enz, Experimental and theoretical study of the domain configuration on thin layers of $\mathrm{BaFe}_{12} \mathrm{O}_{19}$, Philips Res. Rep. 15 (1960), 7-29.

11. L. D. Landau, E. M. Lifshitz, and L. P. Pitaevskii, Statistical Physics, part 2, Course of Theoretical Physics, vol. 9, Pergamon Press, Oxford-New York, 1980.

12. M. E. Taylor, Partial Differential Equations III, Applied Mathematical Sciences, vol. 117, Springer-Verlag, New York, 1997. MR 1477408 (98k:35001)

13. X. Ye, The Fourier collocation method for the Cahn-Hilliard equation, Comput. Math. Appl. 44 (2002), no. 1-2, 213-229. MR 1908282(2003e:65185)

Department of Mathematics, Humboldt-Universität zu Berlin, Unter den Linden 6, D-10099 Berlin, Germany

E-mail address: condette@mathematik.hu-berlin.de

Department of Mathematics I, RWTH Aachen University, D-52056 Aachen, Germany

E-mail address: melcher@math1.rwth-aachen.de

Mathematical Institute, University of Oxford, 24-29 St Giles', Oxford OX1 3LB, United Kingdom.

E-mail address: endre.suli@maths.ox.ac.uk 